\title{
Effects of downregulation of SIRT3 expression on proliferation and apoptosis in esophageal squamous cell carcinoma EC9706 cells and its molecular mechanisms
}

\author{
Mei Yang, Chunsong Yang and Yuhua Pei* \\ Wuhan City Vocational College,10 West Yezhi Lake Road, Mahu Village, Hongshan District, Wuhan \\ 430064, China
}

\begin{abstract}
To investigate the effect of downregulation of SIRT3 expression on cell proliferation and invasion in esophageal squamous cell carcinoma (ESCC) EC9706 cells, and to explore its possible molecular mechanisms, we transfected siRNA targeting SIRT3 into EC9706 cells, and then divided cells into three groups: untreated, control siRNA and SIRT3 siRNA groups. The expression levels of SIRT3 protein were detected in different groups by western blotting. The effect of SIRT3 siRNA on cell proliferation was investigated using the CCK-8 kit. The changes of cell apoptosis were examined by flow cytometry. Finally, the expression levels of cell proliferation and apoptosis related proteins such as p21, Bcl-2 and Bax were determined by western blotting. SIRT3 siRNA effectively down-regulated the expression of SIRT3 protein in EC9706 cells, and the reduced expression of SIRT3 significantly inhibited cell proliferation and induced cell apoptosis. Most notably, the SIRT3 depletion markedly increased the expressions of $\mathrm{p} 21$ and Bax proteins but reduced Bcl-2 protein expression. The proliferation inhibition and apoptosis of EC9706 cells mediated by SIRT3 downregulation may be closely associated with the expression levels of $\mathrm{p} 21, \mathrm{Bcl}-2$ and Bax proteins.
\end{abstract}

Key words: Esophageal squamous cell carcinoma; SIRT3; small interfering RNA; proliferation; apoptosis

\section{Introduction}

Sirtuins (SIRTs) are a class of type III histone deacetylase enzymes, including seven members (SIRT1-7). They harbor various enzymatic activities and are closely related to a wide range of cellular physiological, biochemical and pathological processes such as metabolism, longevity, aging, cancer and stress reaction [1,2]. These proteins contain an evolutionally conserved core domain of SIRT, including a catalytic domain and a NAD+ binding region [1]. Among these members, SIRT3 is an important mitochondrial protein and a deacetylase, which plays a critical role in mitochondrial stress reaction $[2,3]$.

\footnotetext{
${ }^{*}$ Corresponding author: Yuhua Pei, Wuhan City Vocational College, 10 West Yezhi Lake Road, Mahu Village, Hongshan District, Wuhan 430064, China. Tel.: +86 13627140151; E-mail: pei0623@163.com.
} 
Previous studies demonstrated that SIRT3 played important roles in diverse regulation functions of mitochondria, including metabolism, ATP production and the response to oxidative stress [4-6]. In recent years, more and more evidence has indicated that SIRT3 is closely associated with the initiation and development of tumors [7-9]. In different tumors, it may act as a tumor oncogene [10] or a tumor suppressor [11]. Moreover, it has been reported that SIRT3 is highly expressed in esophageal carcinoma and its high expression is related to the short survival time of esophageal cancer patients [12]. However, its molecular mechanisms are still unclear. In the present study, we aimed to investigate the possible roles of SIRT3 in esophageal squamous cell carcinoma (ESCC) and the related molecular mechanisms. siRNA technique was used to down regulate the mRNA and protein expression of SIRT3 in ESCC EC9706 cells and consequently studied the effects of reduced SIRT3 expression on ESCC cell proliferation and apoptosis. We further investigated its possible molecular mechanisms. The study may provide novel experimental evidence for ESCC gene therapy using SIRT3 as a target.

\section{Materials and methods}

\subsection{Experimental materials}

ESCC EC9706 cells were purchased from the cell library of Institute of Biochemistry and Cell Biology, SIBS, CAS. RPMI 1640 medium, trypsin and fetal calf serum were all purchased from Gibco Company (USA). Cell lysates were purchased from Takara Biotechnology Co. Ltd. Lipofectamine 2000 was purchased from Invitrogen (USA). The CCK-8 reagent was from Beyotime Institute of Biotechnology. SIRT3 siRNA, control siRNA and anti-SIRT3, p21, Bcl-2, Bax and $\beta$-actin antibodies were all purchased from Santa Cruz (USA).

\subsection{Cell culture, transfection and grouping of ESCC EC9706 cells}

ESCC EC9706 cells were cultured in RPMI 1640 medium with $10 \% \mathrm{FBS}$ at $37^{\circ} \mathrm{C}$ in a humidified incubator with $5 \% \mathrm{CO}_{2}$. Cells were seeded and logarithmically grew to $90 \%$ confluent. Then, Lipofectamine 2000 was used to accomplish transient transfection. SIRT3 or control siRNAs were transfected into EC9706 cells. After transfection, cells were divided into 3 groups, untreated group (cells without any treatment), control siRNA group (cells were transfected with control siRNA) and SIRT3 siRNA group (cells were transfected with SIRT3 siRNA).

\subsection{Detection of cell proliferation using the CCK-8 kit}

EC9706 cells from untreated, control siRNA and SIRT3 siRNA groups were collected at $24 \mathrm{~h}, 48 \mathrm{~h}$, $72 \mathrm{~h}$ and $96 \mathrm{~h}$ after transfection. For cell proliferation detection, fresh medium with 10\% CCK-8 was added into the test wells and cells were further cultured at $37^{\circ} \mathrm{C}$ for $2 \mathrm{~h}$. A microplate reader was used to obtain the absorbance at $450 \mathrm{~nm}$.

\subsection{Determination of apoptosis by flow cytometry}

EC9706 cells $\left(1 \times 10^{6} / \mathrm{ml}\right)$ from three groups were collected $48 \mathrm{~h}$ after transfection and washed with pre-cold PBS. $100 \mu \mathrm{l}$ cells were taken and put into a test tube. $5 \mu \mathrm{l}$ of Annexin V-FITC and PI 
(Propidium Iodide) was added into the tube, respectively. The tubes were incubated at dark for $15 \mathrm{~min}$. $1 \times 10^{4}$ cells were detected by flow cytometry, and the CellQuest software was applied to apoptosis analysis.

\subsection{Determination of protein expression by western blotting}

EC9706 cells from each group were collected $48 \mathrm{~h}$ after transfection. Cell lysate was used for total protein extraction. Proteins were then loaded into a SDS-PAGE gel for electrophoresis. The proteins were then transferred to a $\mathrm{NC}$ membrane. The membrane was blocked with $5 \%$ skimmed milk in $1 \mathrm{x}$ TBST for $2 \mathrm{~h}$. Anti-SIRT3, p21, Bcl-2, Bax and $\beta$-actin (1:200) primary antibodies were added to the solution, respectively. The membrane was incubated at $4^{\circ} \mathrm{C}$ overnight. A secondary antibody was then added and the membrane was incubated at RT for $2 \mathrm{~h}$. ECL (1-3 min) was used to develop signal. The image was acquired using routine darkroom development techniques. $\beta$-actin was used as an internal control.

\subsection{Statistical analysis}

The SPSS 13.0 software was used to analyze all obtained data. The data were expressed as "mean \pm standard deviation". One-way ANOVA was applied to analyze the differences between groups. $P<$ 0.05 indicated significant difference.

\section{Results}

\subsection{SIRT3 siRNA down regulates the protein expression of SIRT3 in ESCC EC9706 cells significantly}

To investigate the SIRT3 protein level in various treatment ESCC EC9706 cells, Western blotting was utilized to detect the SIRT3 protein expression. The results showed that there was no difference in protein expression of SIRT3 between untreated and control siRNA groups $(P>0.05)$. However, SIRT3 was significantly reduced in SIRT3 siRNA group $(P<0.05)$ (Figures 1 and 2).

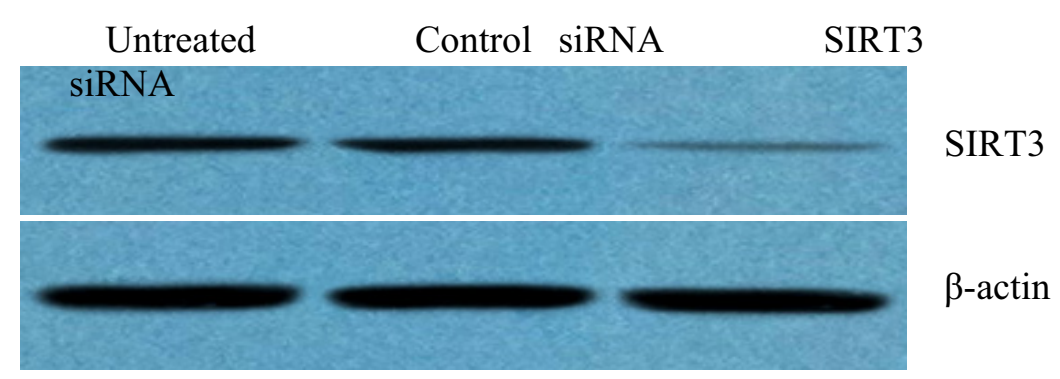

Fig. 1. Detection of SIRT3 protein expression in ESCC cells by western blotting. 


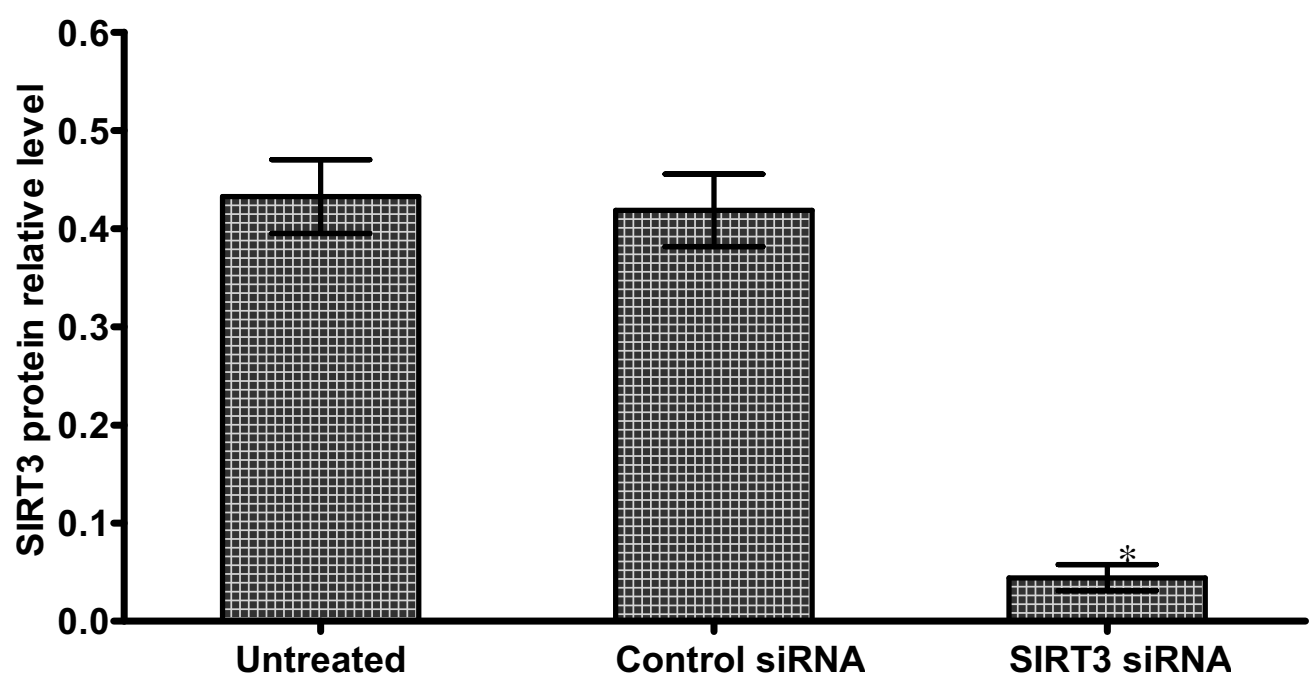

Fig. 2. Relative expression of SIRT3 protein in different ESCC groups. $* P<0.05$, compared to untreated and control siRNA groups

\subsection{The reduced SIRT3 expression inhibits ESCC EC9706 cell proliferation}

The CCK-8 proliferation test indicated that the proliferation of untreated cells and cells transfected with control siRNA was similar $(P>0.05)$. However, compared to untreated and control siRNA groups, the proliferation of ESCC EC9706 cells transfected with SIRT3 siRNA was obviously inhibited and the difference was statistically significant $(P<0.05)$ (Figure 3 ).

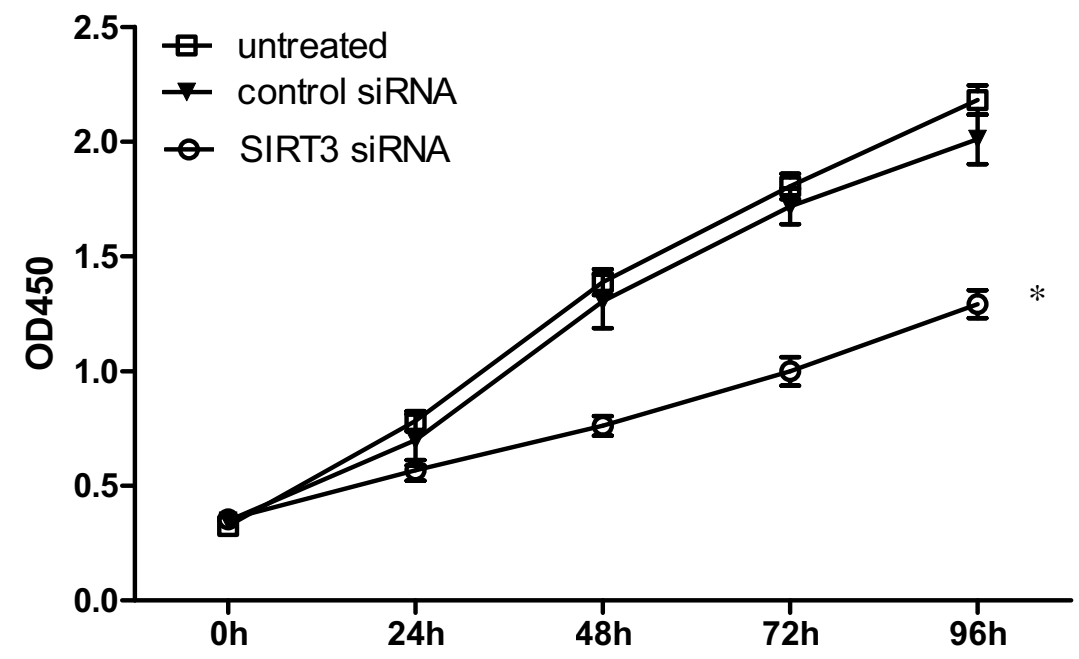

Fig. 3. The reduced SIRT3 expression inhibits ESCC EC9706 cell proliferation. $* \mathrm{P}<0.05$, compared to untreated and control siRNA groups 


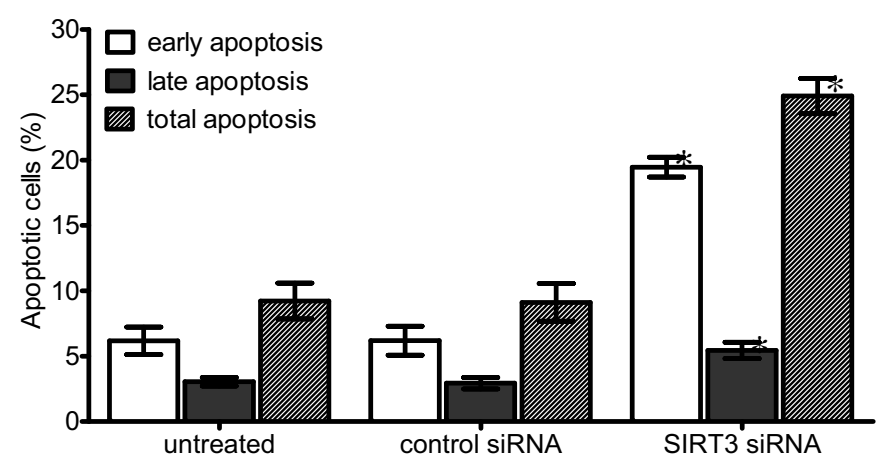

Fig. 4. The effect of reduced SIRT3 expression on ESCC EC9706 cell apoptosis. $* P<0.05$, compared to untreated and control siRNA groups

\subsection{The reduced SIRT3 expression induces ESCC EC9706 cell apoptosis}

According to flow cytometry results, untreated cells and cells transfected with control siRNA had similar early, late and total apoptosis rate $(\mathrm{P}>0.05)$. However, compared to untreated and control siRNA groups, ESCC cells transfected with SIRT3 siRNA showed significantly different early, late and total apoptosis rate $(P<0.05)$ (Figure 4).

\subsection{The effect of reduced SIRT3 expression on apoptosis and invasion related genes in EC9706 cells}

In order to investigate the possible molecular mechanisms for SIRT3-mediated inhibition of proliferation and apoptosis in ESCC cells, we used western blotting to detect the expression of proliferation and apoptosis related proteins like $\mathrm{p} 21, \mathrm{Bcl}-2$ and Bax. The results showed that the expression levels of $\mathrm{p} 21, \mathrm{Bcl}-2$ and Bax proteins were similar between the untreated and control siRNA groups $(\mathrm{P}>0.05)$. However, compared to the untreated and control siRNA groups, the expression of p21 and Bax proteins in cells transfected with SIRT3 siRNA was dramatically increased, while $\mathrm{Bcl}-2$ expression was decreased. All these changes were statistically significant $(\mathrm{P}<0.05)$ (Figures 5 and 6).

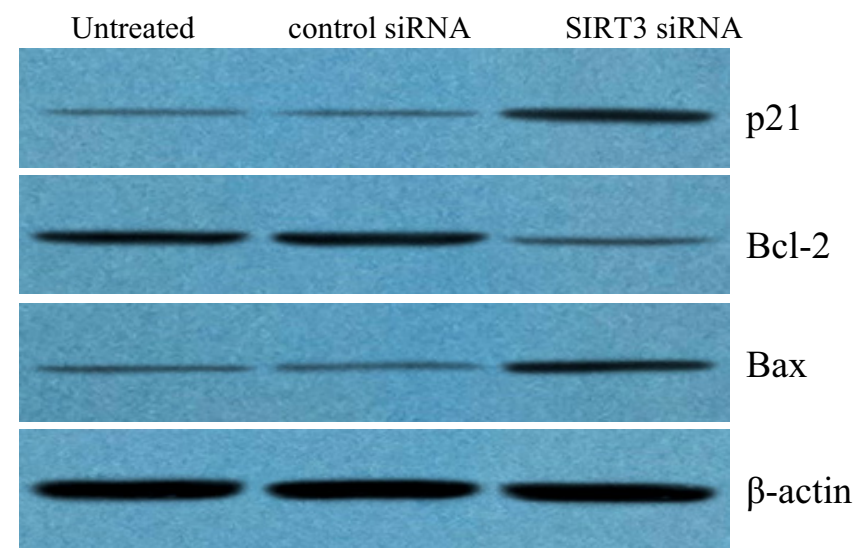

Fig. 5. The expression levels of proliferation and apoptosis related proteins (p21, Bcl-2 and Bax) in ESCC cells with different treatment. Western blotting was used for the detection. 


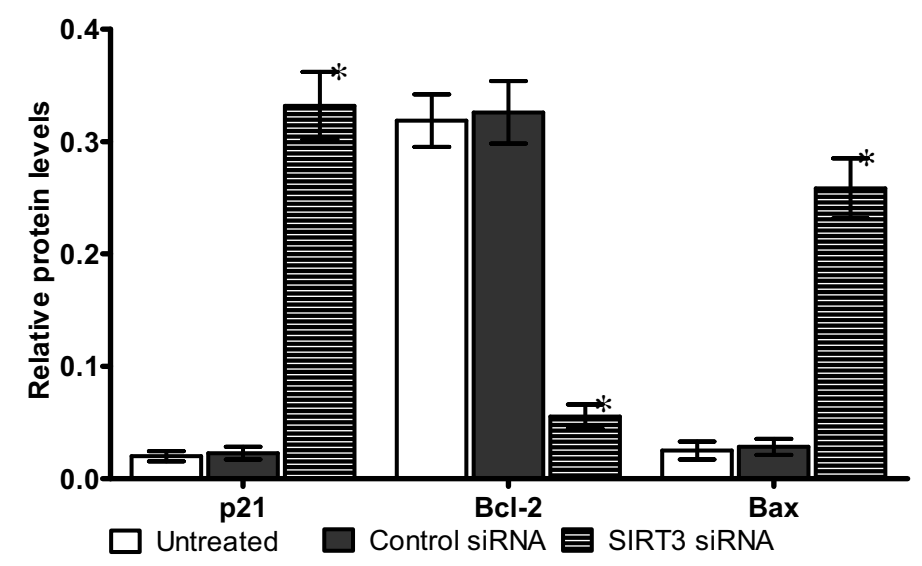

Fig. 6. Relative expression of $\mathrm{p} 21, \mathrm{Bcl}-2$ and Bax proteins in ESCC cells with different treatment. $* P<0.05$, compared to the untreated and control siRNA groups

\section{Discussion}

Currently, SIRT3 has gained intense attention in the cancer field. Its function is considered to be related to cancer [13]. Tumor has six main features, including continuous proliferation signals, insensitivity to anti-proliferation signals, resistance to apoptosis, continuous angiogenesis, unlimited replicative potential and tissue invasion and metastasis [14]. SIRT3 plays an important role in maintaining mitochondrial integrity and function. It can regulate cell survival, apoptosis and metabolism. In addition, it is good for controlling the balance between healthy and disease. Moreover, various studies implicate that SIRT3 has a complex relationship with tumor. SIRT3 expression is obviously higher in oral cancer cells and tissues than in normal control samples. Its down-regulated expression significantly inhibits oral cancer cell proliferation and enhances cytotoxicity of chemotherapy drugs, suggesting that it mainly acts as an oncogene. On the contrary, kaempferol treatment inactivates the Akt signaling pathway and activates apoptosis in chronic myeloid leukemia K562 and acute promyelocytic leukemia U937 cells. The treatment also increases Bax and SIRT3 expression, indicating that SIRT3 acts as a tumor suppressor [15]. Although current studies implicate that SIRT3 expression is significantly higher in esophageal cancer tissues than in normal esophageal tissues, the complex functions of SIRT3 forces us to further study the role of SIRT3 in ESCC so that we may be able to provide new experimental evidence for ESCC gene therapy using SIRT3 as a target.

Research shows that as a new tumor marker, SIRT3 has close relationship with tumor cell proliferation. $\mathrm{Li} \mathrm{H}$ et al. revealed that the reduced SIRT3 level significantly inhibits the acetylation of NMNAT2, the activity of NAD + synthesis and the proliferation of non small cell lung cancer [16]. Furthermore, studies on liver cancer show that SIRT3 is down regulated in hepatocellular carcinoma tissues. Adenovirus-mediated overexpression of SIRT3 obviously inhibits the proliferation of hepatocellular carcinoma cells, reduces intracellular NAD+ level, inhibits the ERK1/2 signaling pathway and activates the Akt/JNK signaling pathway [17]. These results implicate that SIRT3 has the potential to control tumor cell proliferation. In order to further illuminate the regulatory role of SIRT3 in ESCC cell proliferation, we used RNA interference technology to transfect SIRT3 siRNA into ESCC EC9706 cells and revealed that $48 \mathrm{~h}$ after transfection, both mRNA and protein levels of SIRT3 in cells transfected with SIRT3 siRNA were significantly lower than in untreated cells and cells 
transfected with control siRNA. Then, we further used CCK-8 technique to analyze ESCC proliferation at different time points after cells were transfected with SIRT3 siRNA. The results showed that cell proliferation was obviously inhibited 48h-96h after transfection. Notably, p21 expression was dramatically upregulated $48 \mathrm{~h}$ after SIRT3 siRNA transfection. All these data indicate cell proliferation mediated by down regulated SIRT3 expression is very likely related to p21 expression. However, the detailed molecular mechanisms still need to be further investigated.

More and more studies implicate that SIRT3 plays a critical role in mediating tumor cell apoptosis. Xiao K et al. discovered that in A549 lung cancer cells, SIRT3 overexpression induces cell apoptosis, increases the ratio of $\mathrm{Bax} / \mathrm{Bcl}-2$ and $\mathrm{Bad} / \mathrm{Bcl}-\mathrm{XL}$, improves translocation of $\mathrm{AIF}$ into the nucleus, upregulates p53 and p21 expression and downregulates the ROS level. Hence, SIRT3 plays as a tumor suppressor in the initiation and development of lung cancer [18]. In oral squamous cell carcinoma, SIRT3 has the anti-anoikis and anti-tumorgenesis functions. Its high expression mainly affects the CD95/Fas-mediated signaling pathway in oral squamous cell carcinoma [19]. Our results showed that inhibition of SIRT3 expression significantly induced ESCC cell apoptosis, and the reduced SIRT3 expression obviously enhanced Bax protein expression and decreased $\mathrm{Bcl}-2$ protein expression. It suggests that SIRT3-mediated ESCC cell apoptosis may be closely associated with the increased Bax expression as well as the reduced Bcl-2 expression.

Overall, we showed that SIRT3 siRNA significantly down-regulated both mRNA and protein expression of SIRT3 in ESCC EC9706 cells. The reduced expression obviously inhibited ESCC cell proliferation and induced cell apoptosis. The changes may be closely associated with the altered expression of proliferation and apoptosis related proteins. The role of SIRT3 in ESCC and the detailed molecular mechanisms need to be further investigated in the future and the discoveries may provide a theoretical basis for molecular target therapy in ESCC patients.

\section{References}

[1] H.J. Weir, J.D. Lane and N. Balthasar SIRT3: A central regulator of mitochondrial adaptation in health and disease, Genes Cancer 4 (2013), 118-124.

[2] W.C. Hallows, B.N. Albaugh and J.M. Denu, Where in the cell is SIRT3?--functional localization of an NAD+-dependent protein deacetylase, Biochem. J. 411 (2008), e11-e13.

[3] M. Buler, S.M. Aatsinki, V. Izzi et al., Metformin reduces hepatic expression of SIRT3, the mitochondrial deacetylase controlling energy metabolism, PLoS One 7 (2012), e49863.

[4] W.C. Hallows, S. Lee and J.M. Denu, Sirtuins deacetylate and activate mammalian acetyl-CoA synthetases, Proc. Natl. Acad. Sci. U.S.A. 103 (2006), 10230-10235.

[5] B.H. Ahn, H.S. Kim, S. Song et al., A role for the mitochondrial deacetylase Sirt3 in regulating energy homeostasis, Proc. Natl. Acad. Sci. 105 (2008), 14447-14452.

[6] N.R. Sundaresan, M. Gupta, G. Kim et al., Sirt3 blocks the cardiac hypertrophic response by augmenting Foxo3a-dependent antioxidant defense mechanisms in mice, J. Clin Invest. 119 (2009), 2758-2771.

[7] Y. Chen, L.L. Fu, X. Wen et al., Sirtuin-3 (SIRT3), a therapeutic target with oncogenic and tumor-suppressive function in cancer, Cell Death Dis. 5 (2014), e1047.

[8] K.H. Huang, C.C. Hsu, W.L. Fang et al., SIRT3 expression as a biomarker for better prognosis in gastric cancer, World J. Surg. 38 (2014), 910-917.

[9] T.Y. Alhazzazi, P. Kamarajan, E. Verdin et al., Sirtuin-3 (SIRT3) and the hallmarks of cancer, Genes Cancer 4 (2013), $164-171$.

[10] T.Y. Alhazzazi, P. Kamarajan, N. Joo, et al., Sirtuin-3 (SIRT3), a novel potential therapeutic target for oral cancer, Cancer 117 (2011), 1670-1678.

[11] N. Ashraf, S. Zino, A. Macintyre et al., Altered sirtuin expression is associated with node-positive breast cancer, Br. J. Cancer 95 (2006), 1056-1061. 
[12] Y. Zhao, H. Yang, X. Wang et al., Sirtuin-3 (SIRT3) expression is associated with overall survival in esophageal cancer, Ann. Diagn. Pathol. 17 (2013), 483-485.

[13] L. Guarente, Introduction: Sirtuins in aging and diseases, Methods Mol. Biol. 1077 (2013), 3-10.

[14] D. Hanahan and R.A. Weinberg, Hallmarks of cancer: The next generation, Cell 144 (2011), 646-674.

[15] G. Marfe, M. Tafani, M. Indelicato et al., Kaempferol induces apoptosis in two different cell lines via Akt inactivation, Bax and SIRT3 activation, and mitochondrial dysfunction, J. Cell. Biochem. 106 (2009), 643-650.

[16] H. Li, Z. Feng, W. Wu et al., SIRT3 regulates cell proliferation and apoptosis related to energy metabolism in non-small cell lung cancer cells through deacetylation of NMNAT2, Int. J. Oncol. 43 (2013), 1420-1430.

[17] Y.Y. Zhang and L.M. Zhou, Sirt3 inhibits hepatocellular carcinoma cell growth through reducing Mdm2-mediated p53 degradation, Biochem. Biophys. Res. Commun. 423 (2012), 26-31.

[18] K. Xiao, J. Jiang, W. Wang et al., Sirt3 is a tumor suppressor in lung adenocarcinoma cells, Oncol. Rep. 30 (2013), $1323-1328$.

[19] P. Kamarajan, T.Y. Alhazzazi, T. Danciu et al., Receptor-interacting protein (RIP) and Sirtuin-3 (SIRT3) are on opposite sides of anoikis and tumorigenesis, Cancer 118 (2012), 5800-5810. 